\title{
Receptor-mediated delivery of siRNAs by tethered nucleic acid base-paired interactions
}

\author{
KEXIONG ZHANG, ${ }^{1}$ QIAOQIAO WANG, ${ }^{1}{ }^{\text {YANHUA XIE, }}{ }^{1}$ GIL MOR, ${ }^{1}$ EMANUELA SEGA, ${ }^{2}$ \\ PHILIP S. LOW, ${ }^{2}$ and YINGQUN HUANG ${ }^{1}$ \\ ${ }^{1}$ Department of Obstetrics, Gynecology and Reproductive Sciences, Yale University School of Medicine, New Haven, Connecticut 06511, USA \\ ${ }^{2}$ Department of Chemistry, Purdue University and Purdue Cancer Center, West Lafayette, Indiana 47907, USA
}

\begin{abstract}
We report a new strategy for cell-type-specific delivery of functional siRNAs into cells. The method involves the noncovalent attachment of siRNAs to ligand-conjugated oligodeoxynucleotides via nucleic acid base-paired interactions. The resulting complexes can be directly applied to cells, leading to specific cellular uptake and gene silencing. The method is simple, economical, and can be easily adapted for other cell surface receptors. Here we show the application of this method for the delivery of siRNAs to folate receptor-expressing cells.
\end{abstract}

Keywords: siRNA delivery; gene therapy; folate receptor

\section{INTRODUCTION}

The high specificity and potency of RNA interference (RNAi) makes it an especially attractive tool in gene therapy. Like other forms of gene therapy, however, the application of RNAi therapeutics in the form of small interfering RNAs (siRNAs) faces a major obstacle: cell- and tissue-type-specific delivery. In the past years, a variety of cell surface molecules have been exploited for cell-specific siRNA delivery. For example, the Lieberman group used a recombinant protein composed of the protamine-coding region fused to the $\mathrm{C}$ terminus of the heavy-chain Fab fragment of an ErbB2 antibody to assemble protein-siRNA complexes. These complexes were shown to induce specific gene silencing when systemically delivered to ErbB2expressing cancer cells in a mouse model (Song et al. 2005). Instead of using protamine, Schiffelers et al. (2004) employed the self-assembling PEGylated polycation PEI to condense and protect siRNAs. Decorated on the surface of the formulated nanoparticles were Arg-Gly-Asp (RGD) peptides that have high affinity for the $\alpha \mathrm{V} \beta 3 / \alpha \mathrm{V} \beta 5$ integrins that are overexpressed on the surface of endothelial cells of angiogenic blood vessels (for review, see

Reprint requests to: Yingqun Huang, Department of Obstetrics, Gynecology and Reproductive Sciences, Yale University School of Medicine, 300 George Street, New Haven, CT 06511, USA; e-mail: yingqun. huang@yale.edu; fax: (203) 785-7134.

Article published online ahead of print. Article and publication date are at http://www.rnajournal.org/cgi/doi/10.1261/rna.739308.
Temming et al. 2005). These siRNA-containing nanoparticles were successfully delivered to integrin-expressing tumor neovasculature, leading to inhibition of vascular endothelial growth factor receptor-2 (VEGF R2) expression, and thereby, tumor angiogenesis. Similarly, another group encapsulated siRNAs in cyclodextrin-containing polycation particles that had transferrin as a targeting ligand for delivery to transferrin receptor-expressing tumor cells. Systemic delivery of these siRNAs against the EWS-FLI1 gene product resulted in dramatic inhibition of tumor growth in a murine model of metastatic Ewing's sarcoma (Hu-Lieskovan et al. 2005). Recently, RNA aptamers specific for the prostate-specific membrane antigen (PSMA) have been used to carry siRNA-containing complexes into antigen-expressing cells. When noncovalently coupled to siRNAs via a streptavidin bridge, the aptamer:streptavidin: siRNA complexes were efficiently and specifically taken up by antigen-expressing prostate cancer cells and resulted in targeted gene silencing (Chu et al. 2006). Using a different strategy, McNamara et al. (2006) made aptamer-siRNA chimeras through sequence extension of the aptamer and showed that the chimeras were able to silence two tumorrelated genes in addition to reducing cellular proliferation and inducing apoptosis in PSMA-expressing cells.

Folate receptor (FR) is a membrane-associated glycoprotein that binds the vitamin folic acid with nanomolar affinity and mediates the cellular uptake of folate-associated molecules via endocytosis (Leamon and Low 2001). FR is overexpressed in many cancers of epithelial origin and in 
activated macrophages in many inflammatory diseases, but is rarely expressed in normal cells (Parker et al. 2005, and references therein; Paulos et al. 2006). Thus, FR is a potentially useful target for drug delivery. Indeed, folate-linked radioimaging agents have been used clinically to monitor malignant growth in cancer patients, and folate-conjugated chemotherapeutic drugs, liposomes, gene therapy vectors, antisense oligodeoxynucleotides (ODNs), and siRNA chimeras have shown encouraging specific tumor targeting (Turk et al. 2004, and references therein; Guo et al. 2006). Here, we attach siRNAs to folate-conjugated ODNs that contain complementary sequences to the siRNAs and show that the tethered siRNAs can be delivered to FR-expressing cells to elicit specific and efficient gene silencing.

\section{RESULTS}

\section{The tethering strategy}

As illustrated in Figure 1, the oligodeoxynucleotide (ODN) used to tether the various siRNAs is 17 nucleotides (nt) in length. It contains a randomly chosen sequence not complementary to any known human mRNA. The $5^{\prime}$-end of the ODN is chemically conjugated to a folate molecule capable of specific and high-affinity binding to a folate receptor. The ODN contains a 15-nt "hook" sequence that forms base-paired interactions with the "complementary hook" sequence in the $3^{\prime}$-end extension of the sense strand of the desired siRNA. To prepare the tethered siRNA complexes, the folate-conjugated ODN (F-ODN), together with the sense and the antisense strands of a siRNA, were mixed at an equal molar ratio and annealed by heating and slow cooling in a siRNA annealing buffer. The folate moiety of the siRNA complex (F-ODN:siRNA) is expected to target the complexes to its cognate receptor on the cell surface, allowing receptor-mediated endocytosis to mediate siRNA internalization. We hypothesize that following internalization, the action of a cellular nuclease, such as the endonuclease Dicer or an RNase $\mathrm{H}$ (which specifically cleaves the RNA strand of a DNA/RNA duplex), will promote release of the functional siRNA that, in turn, can enable specific gene silencing.

\section{FR expression of cells}

To explore whether tethered siRNAs can be delivered to FR-expressing cells to inhibit gene expression, it was important to test two appropriate cell types for FR expression: a primary culture of human umbilical vein endothelial cells (HUVECs) and human nasopharyngeal carcinoma KB cells. HUVECs have been widely used in studies related to angiogenesis, as they express high levels of the $\alpha \mathrm{V} \beta 3 / \alpha \mathrm{V} \beta 5$ integrins (for review, see Temming et al. 2005). Interestingly, we found that these cells also appear to express FR. KB cells are commonly used in FR-related research due to their elevated expression of FR (Turk et al. 2004, and references therein). We used immunofluorescence and labeled folate delivery assays to examine FR expression

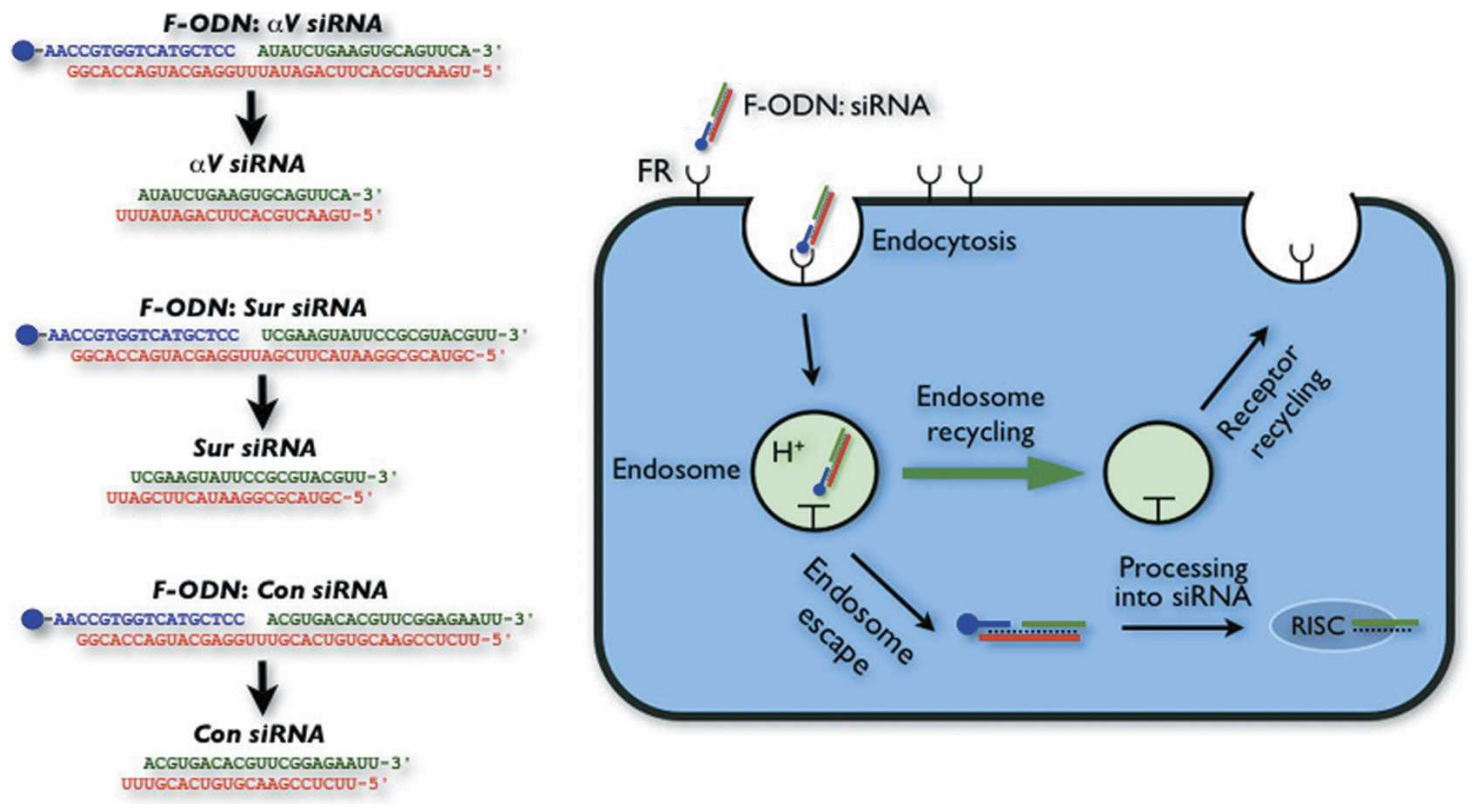

FIGURE 1. Schematic illustration of the tethering strategy and proposed mechanism of action. The siRNAs specific for the human $\alpha \mathrm{V}(\alpha \mathrm{V})$ integrin and survivin (Sur) mRNAs, and a nonsilencing control siRNA used in the studies are shown on the left. The $5^{\prime}$-end of the tethering ODN is covalently conjugated to folate (blue dot). The antisense RNA strands complementary to the target mRNAs are in green. The $3^{\prime}$-ends of the sense RNA strands (red) are extended to form base-paired interactions with the ODN. Following entry into the cell via FR-mediated endocytosis, the siRNA complexes (F-ODN:siRNA) are processed and the released siRNAs incorporated into RNA-induced silencing complex (RISC). 
using MDA-MB-435S human breast cancer cells as a negative control. In the immunofluorescence assay, both HUVECs (Fig. 2A) and KB cells (Fig. 2B) express appreciable levels of FR, while FR expression was undetectable in the breast cancer cells (Fig. 2C). In the functional assay for FR-mediated endocytosis, fluorescence-labeled folate was seen to accumulate in the cytoplasm of the HUVECs (Fig. 2D) and KB cells (Fig. 2E), but not of the breast cancer cells (Fig. 2F). Cytoplasmic fluorescence accumulation was not observed when excess amounts of unlabeled folate were present in the culture medium in the delivery assays (data not shown). Taken together, these results are in agreement with the idea that both HUVECs and KB cells express the FR.

\section{Tethered siRNAs specifically inhibit gene expression}

We chose two genes for testing FR-dependent siRNAmediated gene silencing. One is for the $\alpha \mathrm{V}$ integrin, the subunit of the $\alpha \mathrm{V} \beta 3$ integrin dimer. These molecules are highly expressed on the surface of angiogenic endothelial cells and play critical roles in the process of angiogenesis (for review, see Temming et al. 2005). Inhibition of $\alpha \mathrm{V}$ integrin has been shown to induce endothelial apoptosis, suppress angiogenesis, and increase endothelial monolayer permeability (Qiao et al. 1995; Kumar 2003). The other gene is for survivin, an apoptosis inhibitor that is highly expressed in a variety of malignancies, but minimally expressed in normal cells. As suppression of apoptosis is a hallmark of cancer, development of anticancer strategies that specifically target defects in this pathway is highly desirable.

First, we tested the gene silencing effect of $\alpha \mathrm{VsiRNA}$ on HUVECs. We added the F-ODN: $\alpha \mathrm{V}$ siRNA to the cells at a final concentration of $300 \mathrm{nM}$ in culture medium containing $10 \%$ fetal bovine serum. In parallel, the cells were incubated with buffer alone, F-ODN: $\alpha$ VsiRNA in the

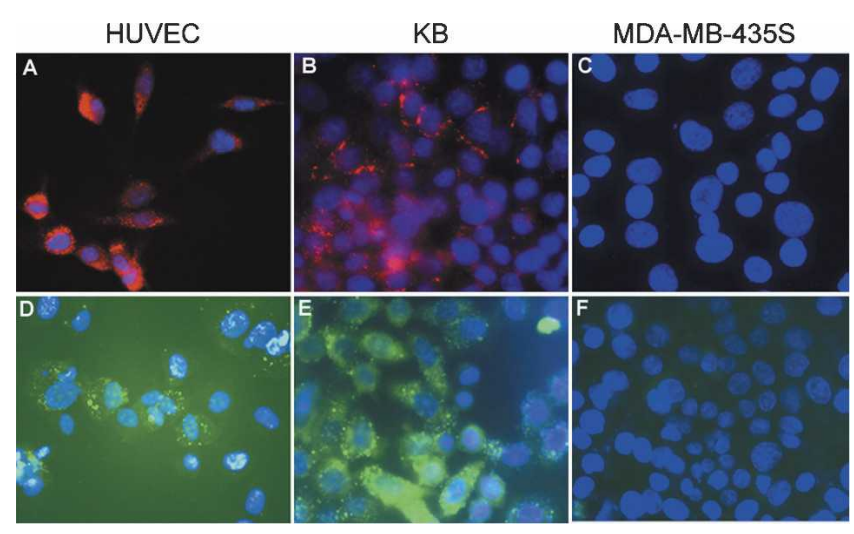

FIGURE 2. FR expression. $(A-C)$ The representative results of immunofluorescence. The anti-FR staining is in red and the nuclear DAPI staining is in blue. $(D-F)$ The representative results of the folate-delivery assay. The green dots indicate cytoplasmic accumulation of folate-oregon green 488 , while the nuclear DAPI staining is in blue. presence of 200-fold molar excess of free folate (to compete for the binding of FR on the cell surface), ODN: $\alpha \mathrm{V}$ siRNA (the tethering ODN did not have folate conjugated), or a nonsilencing F-ODN:Con siRNA. After a $4 \mathrm{~h}$ incubation, the growth medium was changed to fresh HUVEC culture medium containing no siRNAs, and incubation continued for an additional $22 \mathrm{~h}$. To check whether F-ODN: $\alpha \mathrm{V}$ siRNA could specifically reduce target gene expression, total cellular RNAs were extracted and RNA levels measured by quantitative real-time RT-PCR (qRT-PCR). We observed an $\sim 80 \%$ inhibition of $\alpha \mathrm{V}$ mRNA expression in cells treated with F-ODN: $\alpha$ VsiRNA, while the level of the nontargeted $\beta$-actin mRNA was not affected (Fig. 3A). In addition, treatment with the nonsilencing F-ODN:Con siRNA did not reduce the level of $\alpha \mathrm{V}$ mRNA, arguing that the silencing effect is siRNA sequence specific. Since the inhibition was readily competed out by the addition of excess free folate, and since ODN: $\alpha$ VsiRNA failed to produce any inhibitory effect, we conclude that the siRNA delivery is FR mediated. To further verify that the inhibition was indeed dependent on FR, we treated the FR-negative MDA-MB$435 \mathrm{~S}$ cells with the same sets of siRNAs and found no inhibition of gene expression by the $\alpha$ VsiRNA (Fig. 3B).

As siRNA treatment could potentially induce nonspecific inflammatory responses that often lead to an elevated level of interferon- $\beta$ (IFN- $\beta$ ) (Sledz et al. 2003; Kariko et al. 2004 ), we measured the expression of INF- $\beta$ mRNA after the various treatments. Although F-ODN: $\alpha$ VsiRNA induced potent target gene silencing, it did not result in a significant increase in INF- $\beta$ mRNA expression. This was not due to the inability of the cells to the inflammatory responses, because the cells transfected with poly (I:C) produced a two-order-of-magnitude increase in INF- $\beta$ mRNA level (Fig. 3C). The siRNA treatment did not appear to result in nonspecific toxicity to the cells, since neither the cell viability nor the indicated apoptotic enzyme activity was altered (Fig. 3D).

Specific gene silencing effects were also observed with KB cells when a survivin siRNA was used (Fig. 4A), whereas INF- $\beta$ induction and nonspecific cell toxicity were not observed (Fig. 4B; data not shown). The same survivin siRNA delivered to HUVECs also induced specific gene silencing, albeit to a much lesser extent (data not shown). Taken together, these results strongly suggest that the tethered siRNAs cannot only be specifically delivered to FR-expressing cells, but also be processed into siRNAs to generate specific RNAi effects. Since the experiments were performed in the presence of serum, we reason that these siRNA complexes must be reasonably stable against nuclease degradation prior to cellular uptake.

\section{DISCUSSION}

Using our tethering strategy, we have tested two siRNAs that target the $\alpha \mathrm{V}$ integrin and survivin genes for their 
A
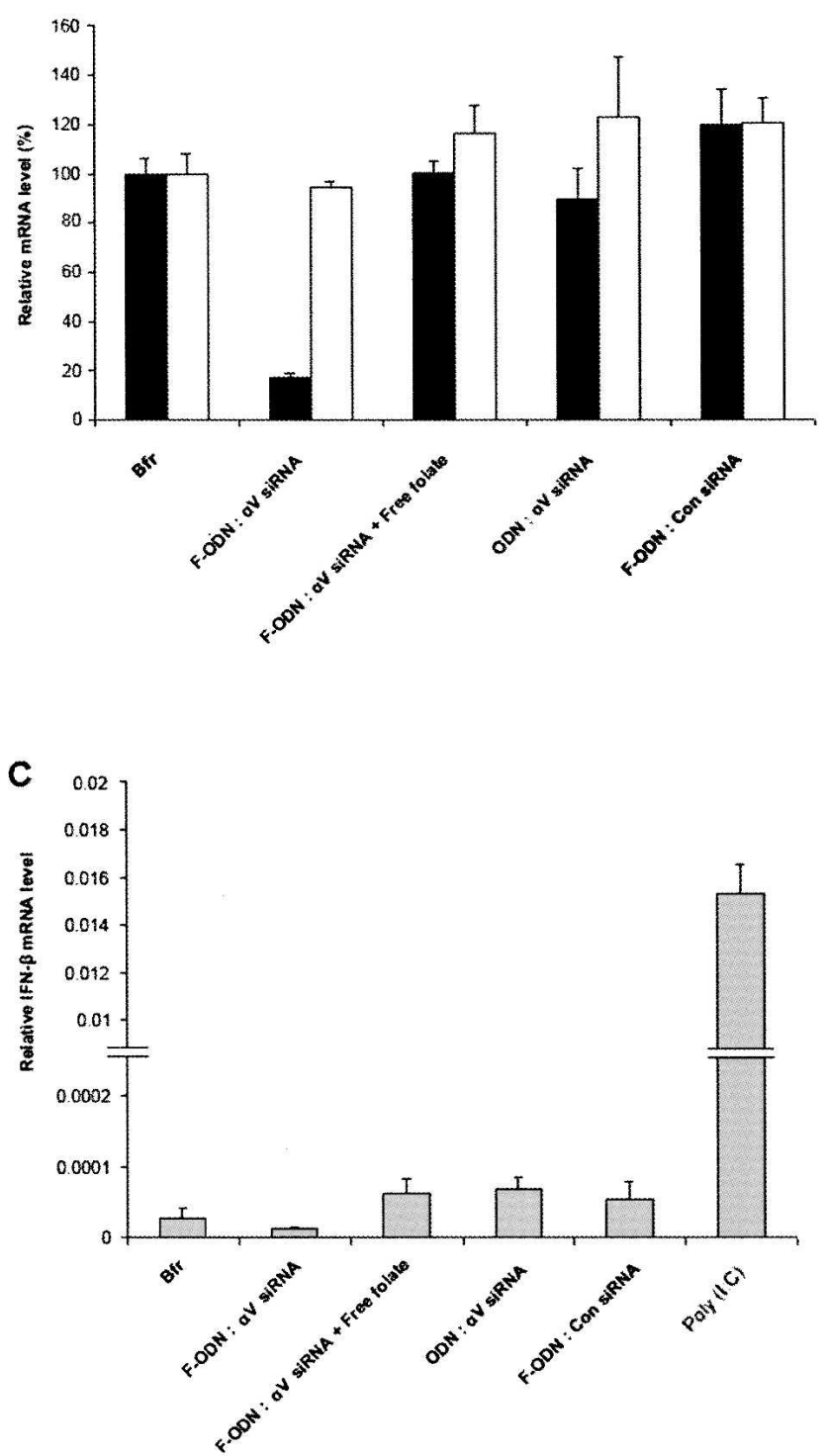

B av mRNA DR-actinmRNA

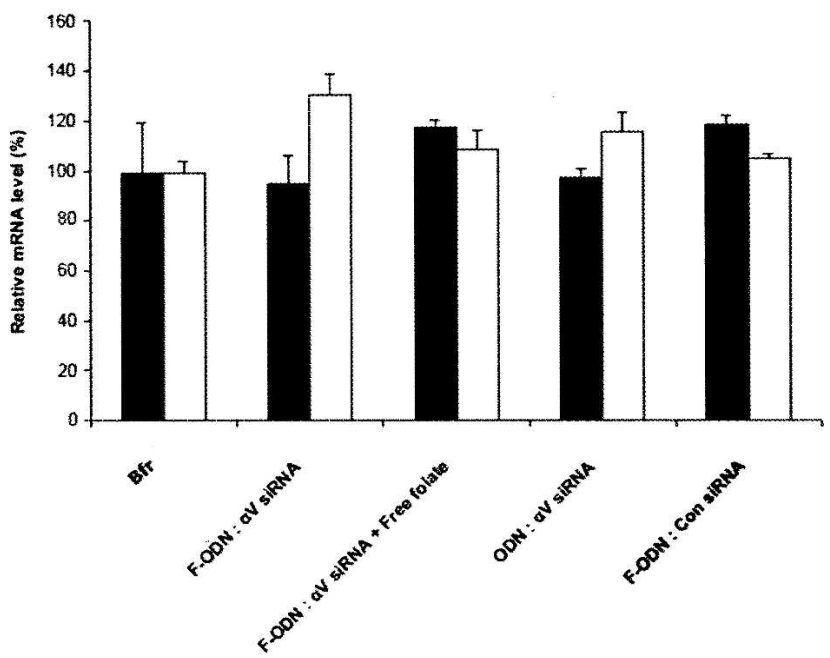

D

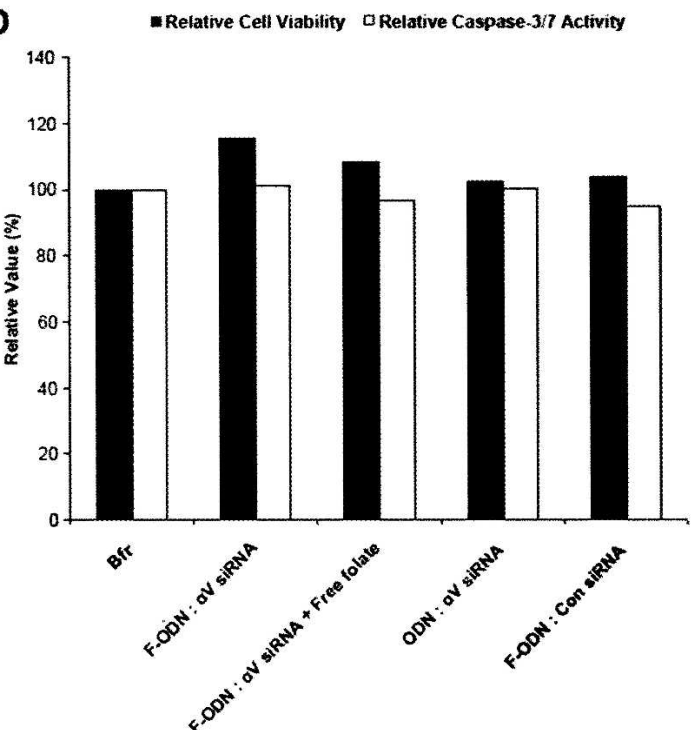

FIGURE 3. Cell-type specific gene silencing by tethered siRNAs. HUVECs $(A, C, D)$ and MDA-MB-435S $(B)$ cells were incubated with buffer alone, or with $300 \mathrm{nM}$ of the various siRNAs in the presence of $10 \%$ fetal bovine serum. Four hours later, the media were removed and cells were incubated in fresh media containing no siRNAs for an additional $22 \mathrm{~h}$. In $C$, last column, HUVECs were transfected with poly (I:C) using Lipofectamine 2000. In A-C, total cellular RNAs were extracted and the indicated mRNA levels measured by qRT-PCR. Each bar represents mean $\pm \operatorname{sem}(n=3-6)$. In $D$, cell viability and caspase activity of HUVECs were measured following the various treatments above, and the results of an average of two independent experiments were plotted. (Bfr) Buffer alone; (F-ODN:siRNA) siRNA tethered by the folate-conjugated ODN; (FODN:siRNA+free folate) F-ODN:siRNA in the presence of 200x molar excess free folate; (ODN:siRNA) siRNA tethered by the same ODN but without folate conjugated; Poly (I:C) cells transfected with poly (I:C).

ability to inhibit gene expression in cells overexpressing the folate receptor. Both siRNAs showed significant and specific gene silencing effects in a folate receptor-dependent manner, but with the survivin siRNA exhibiting somewhat lower potency, possibly because the sequence of the siRNA has not yet been optimized. Based on the fact that two different siRNAs were able to induce specific gene silencing in two different types of cells, we conclude that the method presented here offers the potential to become a general strategy for the delivery of almost any siRNA to almost any FR-expressing cell type.

This method is advantageous in several ways. First, the chemistry involved in ligand-ODN conjugation, synthesis, and purification of ODNs and siRNAs is simple and allows for large-scale production for use in in vivo applications. The simplicity of the method is further underscored by the ease of the procedure by which the tethered siRNA complexes are prepared. In addition, the annealed siRNA 
A

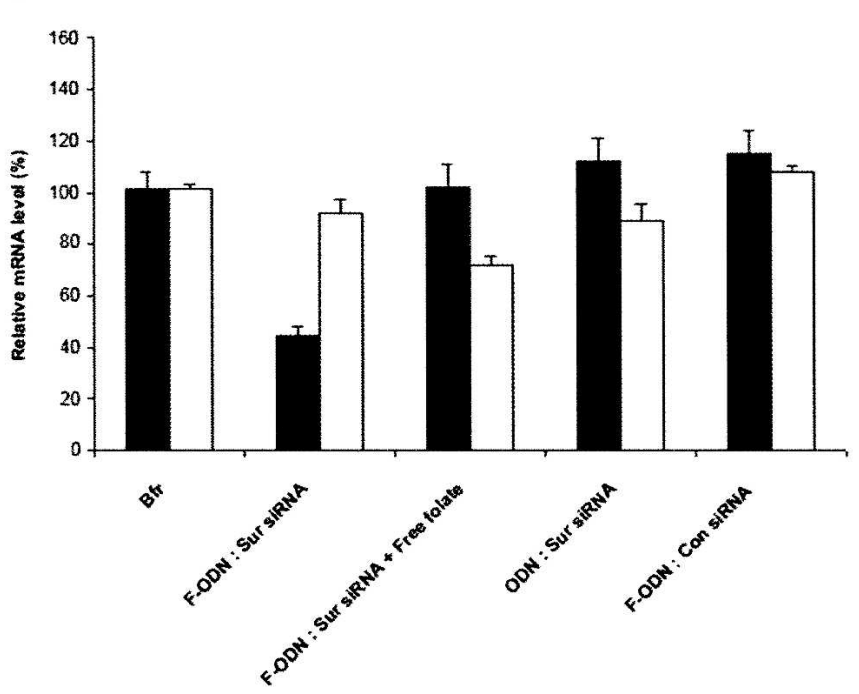

B



FIGURE 4. Specific gene silencing by tethered siRNAs in KB cells. KB cells were incubated with buffer alone, or with $300 \mathrm{nM}$ of the various siRNAs in the presence of $10 \%$ fetal bovine serum. Four hours later, the media were removed and cells incubated in fresh media containing no siRNAs for an additional $22 \mathrm{~h}$. In $B$, last column, KB cells were transfected with poly (I:C) using Lipofectamine 2000. Total cellular RNAs were extracted and the indicated mRNA levels measured by qRT-PCR. Each bar represents mean \pm sem $(n=3-6)$. Other labels are the same as in Fig. 3 .

complexes cannot only be stored frozen, but can also tolerate multiple freeze-thaw cycles without losing gene silencing activity. Further, the individual ODNs and siRNAs should be amenable to various chemical modifications that lead to enhanced nuclease resistance and pharmacokinetics for in vivo applications. Second, the method enables a single conjugated ODN to simultaneously deliver multiple siRNAs specific for different genes or to different regions of a gene to achieve possible synergistic effects, as all siRNAs can be designed to contain the same extended 3 '-end hook sequence. Moreover, the method provides a rapid and easily adaptable solution to targeting cellular uptake of siRNAs to cells expressing other surface antigens. Third, the method has the potential to overcome other prior issues, including poor release of a therapeutic cargo from its covalently attached ligand (Yang et al. 2006). This derives from our underlying hypothesis, not yet demonstrated directly, that the action of Dicer or other cellular nucleases may facilitate the efficient release of a siRNA from its noncovalently attached ligand.

\section{MATERIALS AND METHODS}

\section{Antibodies and DNA and RNA oligos}

The mouse monoclonal antifolate receptor antibody LK26 was purchased from Abcam (ab3361). The tethering ODN with a 5'end free amino group (NH2-ODN) was made by the Yale Keck Facility. The sequence of the ODN: 5'-AACCGTGGTCATG CTCC-3'. HPLC-purified RNA oligos were custom ordered from Integrated DNA Technologies, Inc. $\alpha \mathrm{V}$ sense: 5'-UGAACUGCA CUUCAGAUAUUUGGAGCAUGACCACGG-3'; $\alpha \mathrm{V}$ antisense: $5^{\prime}$ -
AUAUCUGAAGUGCAGUUCA-3' (the core sequences of the siRNA were adapted from Cao et al. 2006); survivin sense: $5^{\prime}$-CG UACGCGGAAUACUUCGAUUGGAGCAUGACCACGG-3'; survivin antisense: 5'-UCGAAGUAUUCCGCGUACGUU-3'; control siRNA sense: 5'-UUCUCCGAACGUGUCACGUUUGGAGCAU GACCACGG-3'; control antisense: 5'-ACGUGACACGUUCGGA GAAUU-3' (the core sequences of the siRNA were adapted from McNamara et al. 2006).

\section{Folate-ODN conjugation}

To conjugate folate to the $5^{\prime}$-end of the tethering ODN, NH2ODN was reacted overnight at room temperature with 10 -fold molar excess folate-NHS (Aronov et al. 2003) dissolved in DMSO in the presence of fivefold molar excess of triethylamine. The resulting folate-ODN was precipitated in cold ethyl ether, resuspended in water, and unconjugated folate removed by gelfiltration chromatography. The conjugate was further purified by resolving on an $8 \%$ denaturing polyacrylamide gel. The conjugation efficiency was estimated as typically $\sim 50 \%-70 \%$ based on polyacrylamide gel analysis. Gel slices containing the conjugate were eluted in elution buffer $(50 \mathrm{mM} \mathrm{NaCl}, 50 \mathrm{mM}$ Tris- $\mathrm{HCl}$ at $\mathrm{pH} 7.5,0.05 \%$ SDS, $10 \mathrm{mg} / \mathrm{mL}$ proteinase $\mathrm{K}$ ) at $4^{\circ} \mathrm{C}$ in dark overnight. The elute was extracted twice with phenol/chloroform, the aqueous phase ethanol precipitated, and the pellet containing the purified conjugate resuspended in nuclease-free water and stored at $-20^{\circ} \mathrm{C}$ until use.

\section{Cell culture and siRNA treatment}

The primary culture of human umbilical vein endothelial HUVECs were obtained from the Yale BCMM core facility. The cells (passage numbers 3-5) were cultured in tissue culture plates coated with $0.1 \%$ gelatin in medium 199 (GIBCO, 11,150), supplemented with $20 \%$ fetal bovine serum, $2.8 \mathrm{mM}$ L-glutamine, 
$5 \mathrm{mg} / 100 \mathrm{~mL}$ of ECGS (endothelial cell growth supplement, freshly added before use) and 10x penicillin-streptomycin (Sigma, P4333$100 \mathrm{~mL}$ ). The human nasopharyngeal carcinoma KB cells (ATCC, CCL-17) and the breast cancer MDA-MB- 435 S cells (ATCC, HTB129) were purchased. Both cells were maintained in folate-free RPMI 1640 medium (GIBCO, 27016-021) supplemented with 10\% fetal bovine serum, which provides a physiological concentration of folate. To prepare for immunofluorescence, HUVECs were seeded in 8-well glass slides (Lab-Tek, 177,402) coated with fibronectin 0.1\% solution (Sigma, F1141-2MG) diluted at 1:1000 in PBS $24 \mathrm{~h}$ before the staining. In the case of $\mathrm{KB}$ and MDA-MB$435 \mathrm{~S}$ cells, the cells were seeded in 8-well glass slides (without fibronectin coating) $72 \mathrm{~h}$ before staining. The cell density before paraformaldehyde fixation was $\sim 90 \%$. For siRNA treatment, cells grown in 96-well plates were incubated with buffer alone (siRNA annealing buffer: $100 \mathrm{mM}$ potassium acetate, $30 \mathrm{mM}$ HEPES at $\mathrm{pH}$ 7.5), or with $300 \mathrm{nM}$ of the various siRNAs in the folate-free RPMI 1640 medium supplemented with $10 \%$ fetal bovine serum. Four hours later, the medium was replaced with fresh medium in the absence of the siRNAs and incubation continued for an additional $22 \mathrm{~h}$, followed by RNA extraction. As a positive control for INF- $\beta$, HUVECs were transfected with poly (I:C) (Amersham Biosciences, 27-4729-01) at $5 \mu \mathrm{g} / \mathrm{mL}$ using Lipofectamine 2000 (Invitrogen). Twenty four-hours after the transfection, RNAs were isolated, followed by qRT-PCR.

\section{RNA extraction and quantitative real-time RT-PCR (qRT-PCR)}

Total cellular RNAs were harvested using the RNeasy mini kit (QIAGEN). Five hundred nanograms of total RNA from each sample was used in reverse transcription (RT) using the iScript cDNA synthesis kit (BioRad). Real-time PCR was carried out on cDNAs using iQ SYBR Green (BioRad) with a DNA Engine Opticon 2 System (MJ Research, Inc.). All reactions were performed in a $25 \mu \mathrm{L}$ volume. Primers for the individual human genes were as follows: gapdh forward: $5^{\prime}$-TTAGCACCCCTGGC CAAGG-3'; gapdh reverse: 5'-CTTACTCCTTGGAGGCCATG-3'; $\alpha \mathrm{V}$ forward: 5'-CGTATCTGCGGGATGAATCT-3'; $\alpha \mathrm{V}$ reverse: $5^{\prime}$-GGGTTGCAAGCCTGTTGTAT-3' (Cao et al. 2006); $\beta$-actin forward: $5^{\prime}$-ATCAAGATCATTGCTCCTCCTGAG-3'; $\beta$-actin reverse: $5^{\prime}$-CTGCTTGCTGATCCACATCTG-3'; survivin forward: 5'-AAAGAGCCAAGAACAAAATTGC-3'; survivin reverse: 5' GAGAGAGAAGCAGCCACTGTTAC-3' (Weikert et al. 2005); INF- $\beta$ forward: $5^{\prime}$-TCCTGTGGCAATTGAATGGGAGGCTTG-3'; INF- $\beta$ reverse: 5'-TGGTCAATGCGGCGTCCTCCTTCTGG-3'. PCR was performed by initial denaturation at $95^{\circ} \mathrm{C}$ for $5 \mathrm{~min}$, followed by $40 \mathrm{cycles}$ of $30 \mathrm{sec}$ at $95^{\circ} \mathrm{C}, 30 \mathrm{sec}$ at $60^{\circ} \mathrm{C}$, and $30 \mathrm{sec}$ at $72^{\circ} \mathrm{C}$. PCR using water instead of template was used as a negative control. Specificity was verified by melting curve analysis and agarose gel electrophoresis. The threshold cycle $(\mathrm{Ct})$ values of each sample were used in the post-PCR data analysis. Gapdh mRNA was used as an internal control for mRNA level normalization.

\section{Indirect immunofluorescence}

Cells grown in 8-well glass slides were fixed for $20 \mathrm{~min}$ in $3 \%$ paraformaldehyde in TBS $(20 \mathrm{mM}$ Tris- $\mathrm{HCl}$ at $\mathrm{pH} 7.4,225 \mathrm{mM}$ $\mathrm{NaCl}$ ) and washed with TBS for $15 \mathrm{~min}$. Autoimmunofluorescence was inhibited by treatment with $0.1 \%$ sodium borohydride in TBS for $40 \mathrm{~min}$. Cells were permeabilized for $5 \mathrm{~min}$ in $1 \%$ SDS in TBS and washed with TBS for $15 \mathrm{~min}$. Nonspecific sites were blocked by incubation with blocking solution (10\% BSA and 10\% goat serum in TBS) for $1 \mathrm{~h}$. Cells were then incubated with the antiFR antibody at 1:500 dilution in the blocking solution at room temperature for $1 \mathrm{~h}$. For negative control staining, pre-immune mouse IgG instead of anti-FR was used. Following incubation, the cells were washed six times for 5 min each with TBS, and stained for $1 \mathrm{~h}$ in the dark with Texas red-conjugated secondary antibodies (Molecular Probe) diluted at 1:500. Following washing with TBS for $15 \mathrm{~min}$, cells were counter-stained with DAPI, washed with TBS for $15 \mathrm{~min}$, rinsed once with $\mathrm{ddH}_{2} \mathrm{O}$, mounted with Vectorshield (Vector), and visualized with a Zeiss immunofluorescence microscope. For antibody specificity, the anti-FR was replaced with pre-immune IgG during immunofluorescence.

\section{Cell viability and caspase-3/7 assays}

Cell viability and caspase activity were determined using the ApoONE Homogeneous Caspase-3/7 Assay (Promega) and the CellTiterBlue Cell Viability Assay (Promega), respectively, according to the manufacturer's protocols.

\section{Fluorescence-labeled folate delivery assay}

Cells grown in 8-well glass slides were incubated with folateoregon green 488 at a final concentration of $200 \mathrm{nM}$ in the folatefree RPMI 1640 media supplemented with 10\% fetal bovine serum in the absence or presence of 500-fold molar excess of unlabeled folate (to compete off the binding of labeled folate). Four hours later, the media were removed and cells fixed with $3 \%$ paraformaldehyde in TBS for $20 \mathrm{~min}$. After washing in TBS for $15 \mathrm{~min}$, cells were permeabilized for $5 \mathrm{~min}$ in 1\% SDS in TBS and washed in TBS for $15 \mathrm{~min}$. Then, cells were stained with DAPI, washed in TBS for $15 \mathrm{~min}$, rinsed once with $\mathrm{ddH}_{2} \mathrm{O}$, mounted with Vectorshield, and visualized with a Zeiss immunofluorescence microscope.

\section{ACKNOWLEDGMENTS}

This work is supported by the Fannie E. Rippel Foundation and Connecticut Department of Public Health Award S01237 to Y.H. The experiments were performed by K.Z., Q.W., Y.X., and E.S.; Y.H. designed and performed the experiments, analyzed the data, and wrote the manuscript; and P.S.L and G.M. provided input into the design of experiments and interpretation of results.

Received July 18, 2007; accepted December 5, 2007.

\section{REFERENCES}

Aronov, O., Horowitz, A.T., Gabizon, A., and Gibson, D. 2003. Folate-targeted PEG as a potential carrier for carboplatin analogs. Synthesis and in vitro studies. Bioconjug. Chem. 14: 563-574.

Cao, Q., Cai, W., Li, T., Yang, Y., Chen, K., Xing, L., and Chen, X. 2006. Combination of integrin siRNA and irradiation for breast cancer therapy. Biochem. Biophys. Res. Commun. 351: 726-732.

Chu, T.C., Twu, K.Y., Ellington, A.D., and Levy, M. 2006. Aptamer mediated siRNA delivery. Nucleic Acids Res. 34: 71-76. doi: 10.1093/nar/gkl388.

Guo, S., Huang, F., and Guo, P. 2006. Construction of folateconjugated pRNA of bacteriophage phi29 DNA packaging motor 
for delivery of chimeric siRNA to nasopharyngeal carcinoma cells. Gene Ther. 13: 814-820.

Hu-Lieskovan, S., Heidel, J.D., Bartlett, D.W., Davis, M.E., and Triche, T.J. 2005. Sequence-specific knockdown of EWS-FLI1 by targeted, nonviral delivery of small interfering RNA inhibits tumor growth in a murine model of metastatic Ewing's sarcoma. Cancer Res. 65: 8984-8992.

Kariko, K., Bhuyan, P., Capodici, J., and Weissman, D. 2004. Small interfering RNAs mediate sequence-independent gene suppression and induce immune activation by signaling through toll-like receptor 3. J. Immunol. 172: 6545-6549.

Kumar, C.C. 2003. Integrin avb3 as a therapeutic target for blocking tumor-induced angiogenesis. Curr. Drug Targets 4: 123131.

Leamon, C.P. and Low, P.S. 2001. Folate-mediated targeting: From diagnostics to drug and gene delivery. Drug Discov. Today 6: 44-51.

McNamara II., J.O., Andrechek, E.R., Wang, Y., Viles, K.D., Rempel, R.E., Gilboa, E., Sullenger, B.A., and Giangrande, P.H. 2006. Cell-type-specific delivery of siRNAs with aptamer-siRNA chimeras. Nat. Biotechnol. 24: 1005-1015.

Parker, N., Turk, M.J., Westrick, E., Lewis, J.D., Low, P.S., and Leamon, C.P. 2005. Folate receptor expression in carcinomas and normal tissues determined by a quantitative radioligand binding assay. Anal. Biochem. 338: 284-293.

Paulos, C.M., Varghese, B., Widmer, W.R., Breur, G.J., Vlashi, E., and Low, P.S. 2006. Folate-targeted immunotherapy effectively treats established adjuvant and collagen-induced arthritis. Arthritis Res. Ther. 8: R77. doi: 10.1186/ar1944.
Qiao, R., Yan, W., Lum, H., and Malek, A.B. 1995. Arg-Gly-Asp peptide increases endothelial hydraulic conductivity: Comparison with thrombin response. Am. J. Physiol. 269: 110-117.

Schiffelers, R.M., Ansari, A., Xu, J., Zhou, Q., Tang, Q., Storm, G., Molema, G., Lu, P.Y., Scaria, P.V., and Woodle, M.C. 2004. Cancer siRNA therapy by tumor selective delivery with ligandtargeted sterically stabilized nanoparticle. Nucleic Acids Res. 32: 141-110. doi: 10.1093/nar/gnh140.

Sledz, C.A., Holko, M., de Veer, M.J., Silverman, R.H., and Williams, B.R. 2003. Activation of the interferon system by short-interfering RNAs. Nat. Cell Biol. 5: 834-839.

Song, E., Zhu, P., Lee, S.-K., Chowdhury, D., Kussman, S., Dykxhoorn, D.M., Feng, Y., Palliser, D., Weiner, D.B., Shankar, P., et al. 2005. Antibody mediated in vivo delivery of small interfering RNAs via cell-surface receptors. Nat. Biotechnol. 23: 709-717.

Temming, K., Schiffelers, R.M., Molema, G., and Kok, R.J. 2005. RGDbased strategies for selective delivery of therapeutics and imaging agents to the tumor vasculature. Drug Resist. Updat. 8: 381-402.

Turk, M.J., Waters, D.J., and Low, P.S. 2004. Folate-conjugated liposomes preferentially target macrophages associated with ovarian carcinoma. Cancer Lett. 213: 165-172.

Weikert, S., Schrader, M., Krause, H., Schulze, W., Muller, M., and Miller, K. 2005. The inhibitor of apoptosis (IAP) survivin is expressed in human testicular germ cell tumors and normal testes. Cancer Lett. 223: 331-337.

Yang, J., Chen, H., Vlahov, I.R., Cheng, J.-X., and Low, P.S. 2006. Evaluation of disulfide reduction during receptor-mediated endocytosis by using FRET imaging. Proc. Natl. Acad. Sci. 103: 13872-13877. 

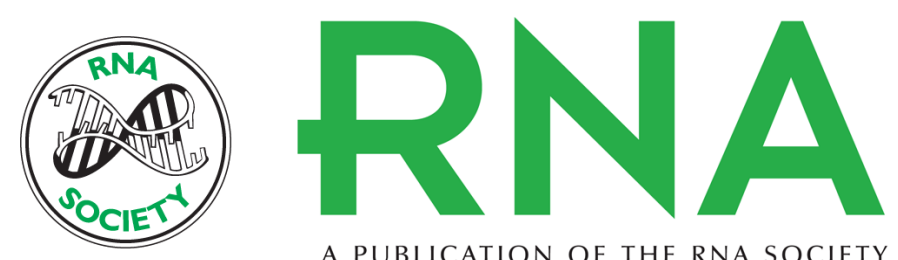

A PUBLICATION OF THE RNA SOCIETY

\section{Receptor-mediated delivery of siRNAs by tethered nucleic acid base-paired interactions}

Kexiong Zhang, Qiaoqiao Wang, Yanhua Xie, et al.

RNA 2008 14: 577-583

References This article cites 19 articles, 3 of which can be accessed free at: http://rnajournal.cshlp.org/content/14/3/577.full.html\#ref-list-1

License

Email Alerting Receive free email alerts when new articles cite this article - sign up in the box at the Service top right corner of the article or click here. 